\title{
Translation Enhancement of m-Rna by Immuno Evasion Proteins
}

\author{
Narmata Arya
}

\begin{abstract}
In this paper, mono-functional EKB i.e. "E3", "K3" and "B18R" derived from vaccinia virus is compared with multi-functional NS1 derived from "influenza $A$ virus" to compare their ability for the enhancement of the mRNA translation. Further, expression of luciferase was transferred by delivery of unmodified luciferase assorted with NS1 mRNA and compared with delivery of unmodified luciferase assorted with either "E3", “K3" or "B18R" respectively. Various combination of "E3", “K3", "B18R" were assorted with NS1-TX91 mRNA at different ratios and delivered with $m$ RNA of luciferace.
\end{abstract}

Keywords: luciferase, NS1 (non-structural protein 1), EKB, mRNA, translation

\section{INTRODUCTION}

Recent clinical trials and preclinical studies have shown excellent potential for mRNA therapy. "Encoding antigens", "transcription factors", "growth factors", "in-vitrotranscribed" (IVT) mRNA can be used as a cancer and infectious disease vaccine or as a "protein replacement therapy", "genome editing" or "cell reprogramming [1].

In- vitro transcribed m-RNA can induce negative response against viruses by stimulating "endosomal" and "pattern recognition receptors" (PRRs), including TLRs (toll like receptors) that includes TLR 3, TLR 8, TLR 7, "protein kinase RNA" (PKR), "retinoic acid-inducible gene I" (RIGI), and "2'-5' oligoadenylate synthetase" (OAS)[2].

In order to improve mRNA translation, an immune evasion approach using viral immune evasion proteins has been suggested. A plethora of "immune evasion proteins" have developed in nature to thwart host antiviral reactions upon viral infection.

By co-delivering "immune evasion proteins" and mRNAof-interest encoding mRNA, immune reactions caused through transfection are efficiently suppressed and translation of m-RNA-of-interest is significantly improved [3]. "EKB" and "NSP1" have been reported to enhance

E3 and K3 inhibit PKR activation, and B18R obstructs "t ype1 IFN signaling" by seizing binding of extracellular IFN to "IFN $\alpha / \beta$ receptor" (IFNAR) on the membrane of the ce 11[4]. NS1 is rather multifunctional in contrast. It inhibits immune-related- proteins such as OAS, PKR, NF-Пb, "interferon regulatory factor 3" (IRF3) as well as "nonimmune-related- proteins" such as "cleavage and factor 30 (CPSF30) polyadenylation"[5].

Binding and inhibiting CPSF30 by NS1 results in accumulation of nonprocessed pre-m-RNA within the nucleus and impedes the expression of host genes, including hundreds of IFN-stimulated genes (ISGs)[6], [7].

Revised Manuscript Received on September 14, 2019.

Narmata Arya, Department of Biotechnology, Sanskriti University, UttarPradesh, India.(E-mail: sanpubip@gmail.com) translation of $\mathrm{m}$-RNA of interest.

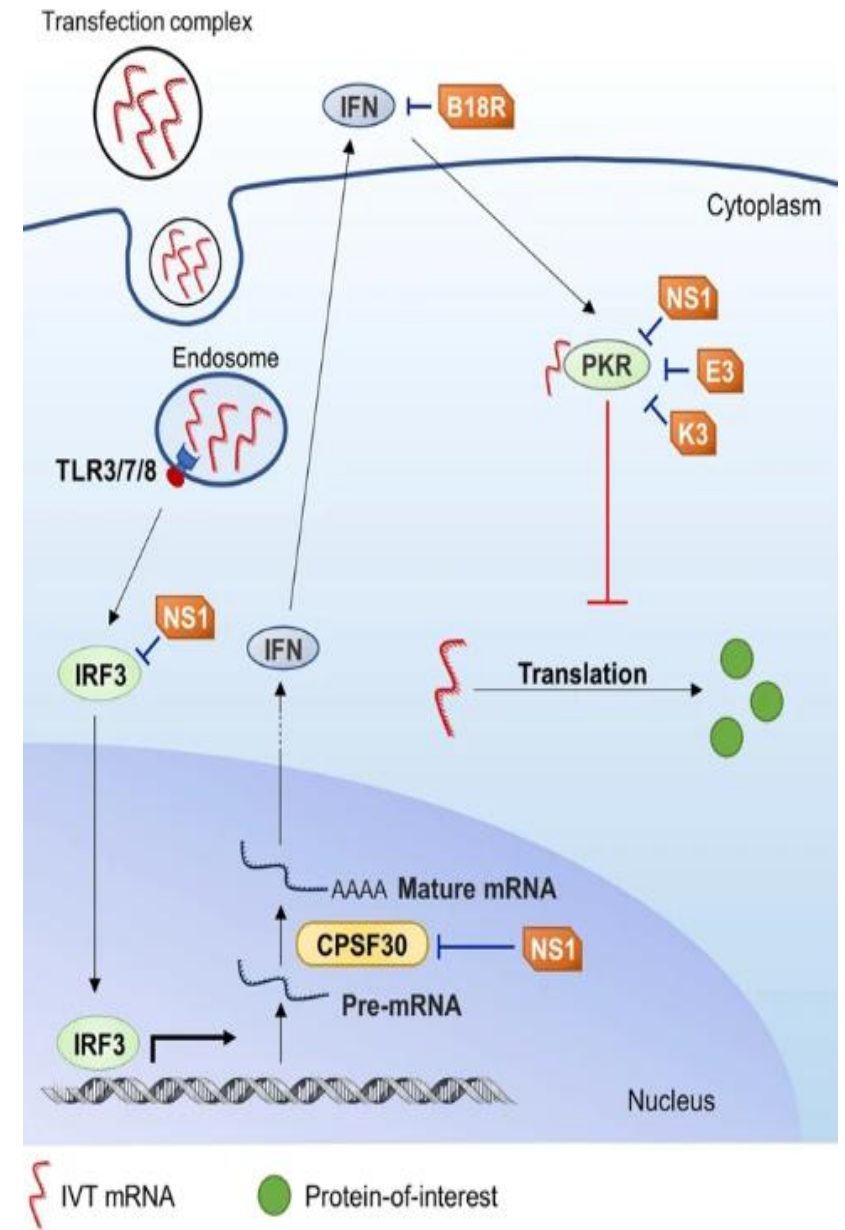

Figure 1: working mechanism of EKB and NS1 in inducing innate immune response

In this research, "EKB" and NS1 with subtype TX91 that is extracted from "strain A / Texas/36/91" were compared on the basis of their capacity to improve mRNA translation. The verification for the effectiveness of these "immune response protein" was done. NS-1 was found more substantial than EKB. Due to lack of supremacy between "NS1-TX9" and EKB, and release of PR8, supremacy of NS1 multifunctionality was established.

\section{MATERIALS}

"Human foreskin fibroblast cell line" that includes "BJ fibroblasts", "ATCC"; "Human hepatocellular carcinoma cell line" that includes "HepG2", "ATCC"; fetal bovine serum (FBS) ;penicillin-streptomycin 100× solution; and trypsin $0.5 \%$ 10× solution; Alamar Blue stock solution ; transfection kit of Stemfect mRNA; "GLO lysis buffer"; "GLO luciferase reagent".

Published By: 


\section{METHOD}

Cloning of EKB genes

Synthesis of EKB genes was carried out by "integrated DNA technologies" (IDT). PCR amplification was done for synthesised genes and further cloned into "Sal-1"and "Not1 " sites of a vector (pGEM4Z-A64).

\section{In-vitro transcription}

Plasmids without B18R gene, were transformed into a linear form with Spe-1. Further it was purified and was used as a template for "in-vitro transcription" by using "T7 High Yield RNA Synthesis Kit" in the presence of an "antireverse cap analog" (ARCA) to produce capped transcripts with approximately 80 percent capping efficiency. Capped transcripts were further used to produce m-RNA modified with pseudouridine. B18R gene containing plasmid were transformed into a linear form with Sac-1.Transformed plasmids were purified and finally transcribed. Polyadenylation of the transcript was performed with E.coli "poly (A) polymerase" (PAP) and confirmed by performing "gel electrophoresis". RNeasy kit was used to purify synthesized m-RNA. Spectrophotometer analysis was carried out for quantification and confirmation of fullsynthesized m-RNA was done by "agarose gel electrophoresis"[8] .

\section{In-vitro transfection}

"Stemfect RNA transfection kit" was used for transfection study[9]. "BJfibroblast" and "HepG2" were placed on a 96 micro-titer plate with a cell density of " $1.2 \times 10^{4 "}$ and was incubated for night. For pseudo modified m-RNA pretreatment, cells were introduced into 40 nanogram of pseudomodified m-RNA. After 6 hours, the fresh medium was added to replace the previous medium and transfected with 40 nanogram of unmodified "luciferase (Luc)" m-RNA. After 18 hours, "Alamar Blue assay" and "Luc assay" were outperformed.

Cells were transfected with "40 nanogram per well of Luc m-RNA and EKB, GFP m-RNA or NS1-TX91 to determine the co-delivery of "immune evasion genes"[10]. To determine the synergism between NS1-TX91 and EKB, transfection of the cells were carried out with Luc $(20 \mathrm{ng})$ and NS1-TX91 (20 ng) alone or in combination of NS1TX91 with "E3", "K3" and/or B18R.

To determine synergism between "E3", "K3", PR8 variants, transfection of the cells were carried out with Luc( $20 \mathrm{ng}$ ) and PR8 mutants (20 ng) alone or in combination with E3, K3, green fluorescent protein (GFP)[11].

\section{Biochemical assay}

Further biochemical assay was carried out to determine the cell viability, and quantify the luciferase expression.

\section{RESULTS}

\section{Translation enhancement}

In this paper it was seen that NS1-TX91 could enhance the m-RNA translation more effectively as compared to EKB.
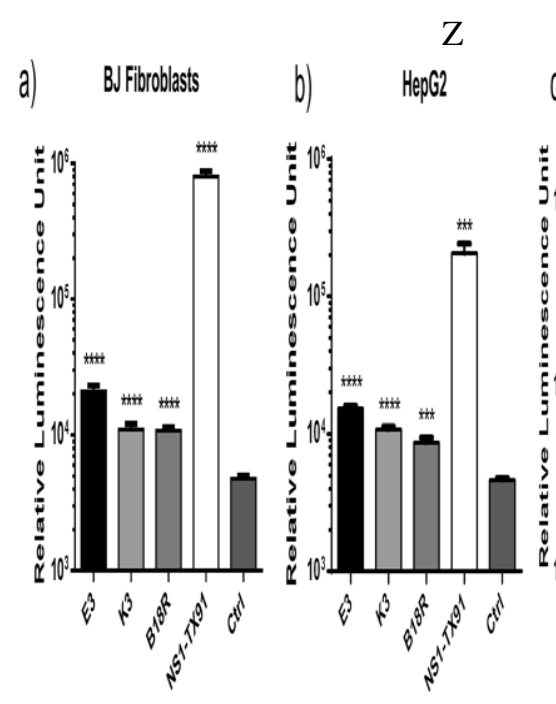

c) HepG

\section{Figure 2: comparison of EKB and NS1-TX91 in translation enhancement}

Figure 2a: comparison of EKB and NS1-TX91 with BJ Fibroblasts,Figure 2b: comparison of EKB and NS1-TX91 with HepG2 cells, Figure 2c: comparison of EKB and NS1TX91 with HepG2 cells for co-delivery "immune responsive protein".

Figure 2 a,b shows that, m-RNA encoding numerous "immune responsive protein" demonstrated higher m-RNA translation of luciferase as compared to "green fluoroscent protein" control.

Figure 2c, demonstrated that, NS1-TX91 showed a 10 fold increase in luciferase production than EKB at different ratios.

\section{Interferon suppression by m-RNA transfection}

Suppression of interferon production was assayed after 18 hours by collecting supernatant of HepG2, and BJ fibroblast and IFN-beta and transfecting witth "EKB", "GFP" or "TX91". Further it was measured by using technique ELISA[12]. It was concluded that "EKB" and "TX91" suppressed immune response more effectively at the time of transfection.

\section{BJ Fibroblasts}

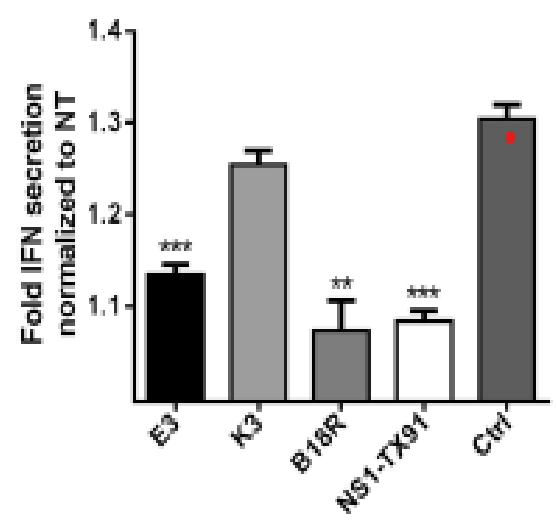

Figure 3a: interferon suppression in BJ Fibroblasts 


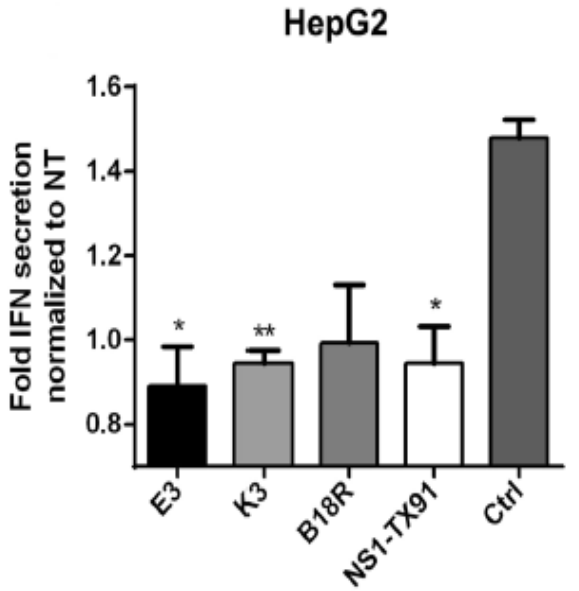

Figure 3b: interferon suppression in HepG2

\section{Host gene expression inhibition}

"Host gene expression inhibition" (HGEI) expression was verified by the co-delivery of HGEI with plasmid luciferase (mutants). Table 1depicts the gain of "host gene expression inhibition" an loss of PKR inhibition.

\begin{tabular}{|c|c|c|c|c|c|c|c|c|c|c|}
\hline \multirow[b]{2}{*}{ Abbreviation } & \multicolumn{6}{|c|}{ Amino adid positions } & \multirow{2}{*}{\begin{tabular}{|l} 
IRF3 \\
R38, \\
K4I
\end{tabular}} & \multirow{2}{*}{$\begin{array}{l}\text { CPSF30 } \\
\text { F103, } \\
\text { M106 }\end{array}$} & \multirow{2}{*}{\begin{tabular}{|l|} 
PKR \\
II23, \\
MI24
\end{tabular}} & \multirow[b]{2}{*}{ Ref } \\
\hline & 38 & 41 & 103 & 106 & 123 & 124 & & & & \\
\hline 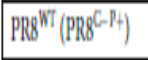 & $R$ & K & s & 1 & 1 & M & Yes & No & Yes & |I \\
\hline PRglliph- & A & A & $\$$ & I & 1 & M & No & No & Yes & "II \\
\hline PRPBRR-(PR8C-P-) & $\mathbb{R}$ & K & s & 1 & A & A & Yes & No & No & "॥ \\
\hline PrgC & $\mathbb{R}$ & K & F & M & A & A & Yes & Yes & No & - \\
\hline 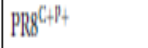 & $\mathbb{R}$ & K & F & M & 1 & M & Yes & Yes & Yes & "॥ \\
\hline
\end{tabular}

\section{Table 1: immune evasion process for HGEI and PKR inhibition}

\section{CONCLUSION}

The potential of viral "immune evasion proteins" i.e. "E3", "K3", "B18R" and "NS1-TX91" was verified to enhane translation of m-RNA. It was concluded that "NS1TX91" showed charecterstic performance in "BJ fibroblast" and "HepG2" as compared to "EKB". Synergism was also not seen between "NS1-TX91" and "EKB". Finally, "EKB" and "NS1" showed translation enhancement but, NS1showed super perferomance in enhancing m-RNA translation.

\section{REFERENCES}

1. D. An et al., "Systemic Messenger RNA Therapy as a Treatment for Methylmalonic Acidemia," Cell Rep., 2017.

2. N. Receptors, R. Hughes, S. Biochemistry, S. Science, and B. Media, "A Handbook of Transcription Factors," Compass, 2011.

3. A. Rustagi and M. Gale, "Innate antiviral immune signaling, viral evasion and modulation by HIV-1," Journal of Molecular Biology. 2014.

4. D. G. Courtney et al., "Epitranscriptomic Enhancement of Influenza A Virus Gene Expression and Replication," Cell Host Microbe, 2017.
5. M. A. Poleganov et al., "Efficient Reprogramming of Human Fibroblasts and Blood-Derived Endothelial Progenitor Cells Using Nonmodified RNA for Reprogramming and Immune Evasion," Hum. Gene Ther., 2015.

6. R. L. Kuo et al., "Interactome Analysis of NS1 Protein Encoded by Influenza A H7N9 Virus Reveals an Inhibitory Role of NS1 in Host mRNA Maturation," $J$. Proteome Res., 2018.

7. F. Ozsolak et al., "Comprehensive polyadenylation site maps in yeast and human reveal pervasive alternative polyadenylation," Cell, 2010.

8. K. K. L. Phua, K. W. Leong, and S. K. Nair, "Transfection efficiency and transgene expression kinetics of mRNA delivered in naked and nanoparticle format," J. Control. Release, 2013.

9. E. V. B. Van Gaal et al., "How to screen non-viral gene delivery systems in vitro?," Journal of Controlled Release. 2011.

10. G. L. Smith et al., "Vaccinia virus immune evasion: Mechanisms, virulence and immunogenicity," Journal of General Virology. 2013.

11. N. S. Scrimshaw and J. P. SanGiovanni, "Synergism of nutrition, infection, and immunity: An overview," American Journal of Clinical Nutrition. 1997.

12. P. J. Tighe, R. R. Ryder, I. Todd, and L. C. Fairclough, "ELISA in the multiplex era: Potentials and pitfalls," Proteomics - Clinical Applications. 2015. 